\title{
Genome Sequence Resource of Pseudomonas fulva HARBPS9.1-Candidate Biocontrol Agent
}

\author{
Adetomiwa A. Adeniji, ${ }^{1}$ Ayansina S. Ayangbenro, ${ }^{2}$ and Du Toit Loots ${ }^{1, \dagger}$ \\ ${ }^{1}$ Human Metabolomics, Faculty of Natural and Agricultural Science, North-West University, Private Bag \\ X6001, Box 269, Potchefstroom, 2531, South Africa \\ ${ }^{2}$ Food Security and Safety Niche Area, Faculty of Natural and Agricultural Science, North-West University, \\ Private Bag X2046, Mmabatho 2735, South Africa
}

\begin{abstract}
The genus Pseudomonas contains a variety of genomic robust strains and species, well known for their beneficial use in a variety of applications, hence the vast amount of research done on this organism to date. We report here the draft genome sequence of an anti-Fusarium rhizospheric Pseudomonas fulva HARBPS9.1 strain from South Africa. This genome analysis identified clusters of genes responsible for the synthesis of pyoverdin and rhizomide in HARBPS9.1; these compounds should confer a competitive advantage on the pseudomonad.
\end{abstract}

Funding

Support was provided to A. A. Adeniji through a post-doctoral fellowship from North-West University.

\section{Keywords}

anti-Fusarium, bioactive metabolites, biocontrol, biological control, disease control and pest management, food safety, fungal pathogens, genome, genomics, microbe-genome sequencing, Pseudomonas, sequencing
The biotechnological benefits ascribed to some genera of bacteria (such as Agrobacterium, Bacillus, Paenobacillus, and Pseudomonas) and their metabolites (antibiotics, lytic enzymes, volatiles, and biosurfactants) are well documented (Berg 2009; Kalia et al. 2017; Meena and Kanwar 2015; Singh et al. 2007). Studies on the pseudomonads specifically, which span over 3 decades, have identified various biomolecules (e.g., amphisin, hydrogen cyanide [HCN] phenazines, 2, 4-diacetylphloroglucinol, pyrrolnitrin, siderophores, tensin, lytic enzymes, rhamnolipids, and viscosinamide) and associated these with many of the beneficial traits for which pseudomonads are well known (Götze and Stallforth 2020; Gross and Loper 2009; Loeschcke and Thies 2015; Zhang et al. 2019). Some members of the genus Pseudomonas, including Pseudomonas chlororaphis, P. fluorescens, P. fulva, P. parafulva, P. putida, and $P$. mosselii, are particularly well known for their antiphytopathogenic (biocontrol and plant growth promoting) properties (Kakembo and Lee 2019; Nielsen et al. 1999; Omoboye et al. 2019; Wu et al. 2018; Zhang et al. 2019), and have comprehensively been investigated for use as possible alternatives to chemical pesticides and synthetic fertilizers used in agriculture (Babalola 2010).

Previously, we identified the anti-Fusarium candidate P. fulva HARBPS9.1, and described its possible use in the in planta management of fusariosis in South Africa (Adeniji et al. 2020). Fusariosis, caused by toxicogenic Fusarium strains (e.g., F. verticilliodes, F. oxysporum, F. culmorum, and $F$. graminearum), affects the distribution of major cereals (such as wheat, barley, and maize) on a global scale (Adeniji and Babalola 2018). In the previous study (Adeniji et al. 2020), P. fulva HARBPS9.1 pure culture was obtained from a distinct colony after a selective isolation was carried out from $5 \mathrm{~g}$ of maize farm rhizospheric soil using Pseudomonas agar (P1852; Sigma-Aldrich) plates with a cetrinix supplement (C8721; Sigma-Aldrich) following the manufacturer's instructions. The in vitro antagonistic property of $P$. fulva HARBPS9.1 (determined on potato dextrose agar; Oxoid) $\left(28^{\circ} \mathrm{C}\right.$ for 7 days $)$ is attributed to the production of bioactive metabolites ( $\mathrm{HCN}$, pyrrolnitrin, and rhamnolipids) and, hence, the isolate was

${ }^{\dagger}$ Corresponding author: D. T. Loots: dutoit.loots@ nwu.ac.za

The author(s) declare no conflict of interest.

Accepted for publication 25 November 2020. 


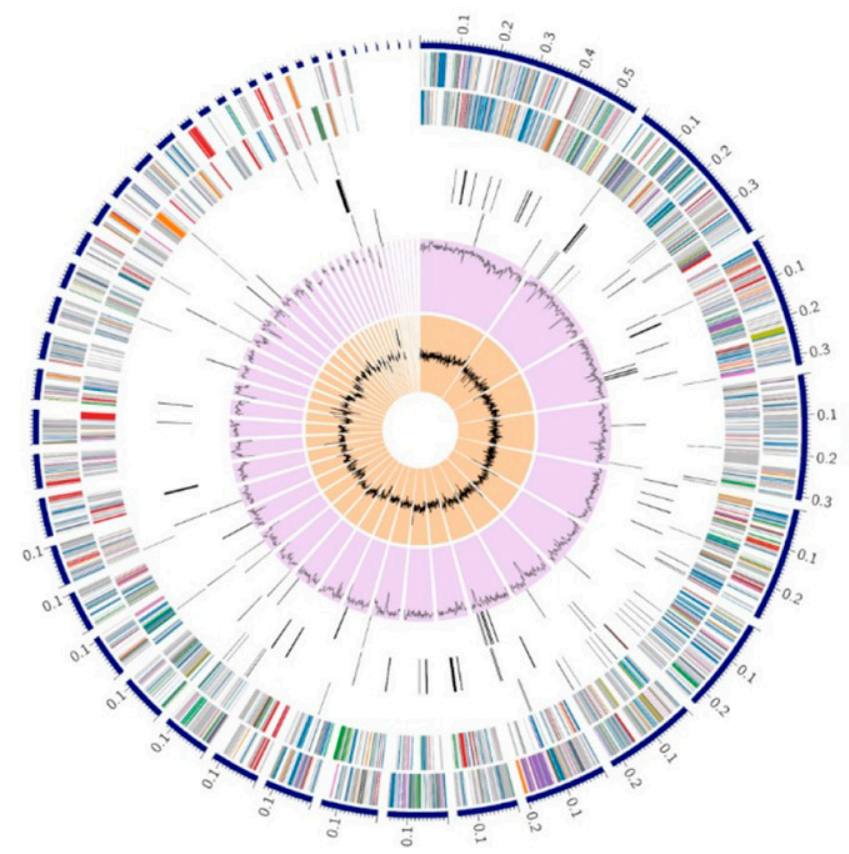

Pseudomonas fulva HARBPS9.1

Subsystem (Subsystems, Genes)

METABOLISM (111, 905)

- PROTEIN PROCESSING $(43,244)$

ENERGY $(29,277)$

- STRESS RESPONSE, DEFENSE, VIRULENCE $(29,137)$

MEMBRANE TRANSPORT $(25,198)$

IINA PROCESSING $(20,103)$

CELLULAR PROCESSES $(17,150)$

RNA PROCESSING $(16,88)$

11. CELL ENVELOPE $(8,68)$

MISCELLANEOUS $(5,21)$

inEGLATION AND CELL SIGNALING $(4,13)$

Fig. 1. Circular atlas of the distribution and subsystem summary of the Pseudomonas fulva HARBPS9.1 genome annotations. The subsystem features from the outer to inner rings include contigs, coding sequence (CDS) (forward strand and reverse strand), RNA genes, CDS for antimicrobial resistance genes and virulence factors, GC content, and GC skew. Genomic features are depicted by the CDS shading on both the forward and reverse strands (Wattam et al. 2017).

molecularly and phylogenetically characterized (16S ribosomal RNA [rRNA] GenBank accession number MF098600.1). Given that very little is currently known about the beneficial properties of the $P$. fulva sp., we sequenced the genome of the isolate $P$. fulva HARBPS9.1 to further identify any novel beneficial traits which it may have.

The genomic DNA of the P. fulva HARBPS9.1 strain was extracted using the Zymo Research ZR Soil Microbe DNA Miniprep Genomic Isolation Kit. The DNA was sequenced with the Illumina MiSeq Reagent Kit v2 microsystem at Molecular Research (MR) DNA, Shallowater, TX, U.S.A., following their in-house protocols. The library was constructed with the Nextera DNA sample preparation kit (Illumina), after which the Qubit double-stranded DNA high-sensitivity assay kit (Life Technologies, Inc.) was used for the final library concentration. The average library size was determined using an Agilent 2100 Bioanalyzer (Agilent Technologies), followed by library pooling and dilution to $12.0 \mathrm{pM}$. The paired-ends sequencing was done using a $600-$ cycle v. 3 reagent kit (Illumina), with an average coverage of 50.0-fold. The Kbase platform (Arkin et al. 2016) was used for checking reads quality (FastQC v.1.0.1), reads trimming (Trimmomatic v0.32), gaps closing, and adaptor sequences removal (Cutadapt v1.0.1). The reads were assembled into contigs using SPAdes v3.13.0 on the KBase platform. The Kbase contigs were then uploaded on the NCBI Prokaryotic Genome Automatic Annotation Pipeline (PGAAP v4.2) (Pruitt et al. 2011), PATRICK online server (v3.3.15) (Wattam et al. 2017), and RAST and SEED online server (version 2.0) (Aziz et al. 2008; Overbeek et al. 2014) for automated annotation and comparison. Default settings were used for all of the bioinformatics analysis.

The sequencing yielded an average read length of $151 \mathrm{bp}$ and 13,137,854 paired-end reads in total. The size of the $P$. fulva HARBPS9.1 genome and number of contigs were $4,822,642$ bp and 47 , respectively, with a $\mathrm{G}+\mathrm{C}$ content of $61.6 \%$. The $\mathrm{N}_{50}$ value of the raw sequences was 203,762 bp and, in total, 4,508 coding genes were annotated, including 7 rRNAs, 63 tRNAs, 4 ncRNAs, and 54 pseudo genes. The subsystem analysis for this genome is shown in Figure 1. Using the antiSMASH (v.5.2) (Blin et al. 2019) default parameters, the biosynthetic clusters (seven clusters) and bioproducts (e.g., pyoverdin and rhizomide) present in $P$. fulva HARBPS9.1 were predicted. The nonribosomal peptide synthetase secondary metabolite rhizomide predicted in the HARBPS9.1 genome sequence makes it a unique pseudomonad and, hence, of interest for further study as to (i) rhizomide's possible beneficial role in HARBPS9.1 antimicrobial activities and (ii) the possible application of HARBPS9.1 for biocontrol. 
This Whole Genome Shotgun project has been deposited at DNA Data Bank of Japan/ European Nucleotide Archive/GenBank under the accession JAATHC000000000; GCA_ 011777485.1 (https://www.ncbi.nlm.nih.gov/assembly/GCA_011777485.1). The version described in this article is version JAASGY010000000. The BioProject and BioSample designations for this project are PRJNA544897 (https://www.ncbi.nlm.nih.gov/bioproject/PRJNA614623) and SAMN11866291 (https://www.ncbi.nlm.nih.gov/biosample/SAMN14435805/), respectively.

\section{Literature Cited}

Adeniij, A. A., Aremu, O. S., Loots, D. T., and Babalola, O. O. 2020. Pseudomonas fulva HARBPS9.1: Candidate anti-Fusarium agent in South Africa. Eur. J. Plant Pathol. 157:767-781.

Adeniji, A. A., and Babalola, O. O. 2018. Tackling maize fusariosis: In search of Fusarium graminearum biosuppressors. Arch. Microbiol. 200:1239-1255.

Arkin, A. P., Stevens, R. L., Cottingham, R. W., Maslov, S., Henry, C. S., Dehal, P., Ware, D., Perez, F., Harris, N. L., Canon, S., et al. 2016. The DOE systems biology knowledgebase (KBase). BioRxiv. doi:10.1101/096354

Aziz, R. K., Bartels, D., Best, A. A., DeJongh, M., Disz, T., Edwards, R. A., Formsma, K., Gerdes, S., Glass, E. M., and Kubal, M. 2008. The RAST Server: Rapid annotations using subsystems technology. BMC Genomics 9:75.

Babalola, O. O. 2010. Beneficial bacteria of agricultural importance. Biotechnol. Lett. 32: 1559-1570.

Berg, G. 2009. Plant-microbe interactions promoting plant growth and health: Perspectives for controlled use of microorganisms in agriculture. Appl. Microbiol. Biotechnol. 84:11-18.

Blin, K., Shaw, S., Steinke, K., Villebro, R., Ziemert, N., Lee, S. Y., Medema, M. H., and Weber, T. 2019. antiSMASH 5.0: Updates to the secondary metabolite genome mining pipeline. Nucleic Acids Res. 47:W81-W87.

Götze, S., and Stallforth, P. 2020. Structure, properties, and biological functions of nonribosomal lipopeptides from pseudomonads. Nat. Prod. Rep. 37:29-54.

Gross, H., and Loper, J. E. 2009. Genomics of secondary metabolite production by Pseudomonas spp. Nat. Prod. Rep. 26:1408-1446.

Kakembo, D., and Lee, Y. H. 2019. Analysis of traits for biocontrol performance of Pseudomonas parafulva JBCS1880 against bacterial pustule in soybean plants. Biol. Control 134:72-81.

Kalia, V. C., Shouche, Y., Purohit, H. J., and Rahi, P., eds. 2017. Mining of Microbial Wealth and Metagenomics. Springer, Singapore.
Loeschcke, A., and Thies, S. 2015. Pseudomonas putida-A versatile host for the production of natural products. Appl. Microbiol. Biotechnol. 99:6197-6214.

Meena, K. R., and Kanwar, S. S. 2015. Lipopeptides as the antifungal and antibacterial agents: Applications in food safety and therapeutics. BioMed Res. Int. 2015:473050.

Nielsen, T., Christophersen, C., Anthoni, U., and Sørensen, J. 1999. Viscosinamide, a new cyclic depsipeptide with surfactant and antifungal properties produced by Pseudomonas fluorescens DR54. J. Appl. Microbiol. 87:80-90.

Omoboye, O. O., Oni, F. E., Batool, H., Yimer, H. Z., De Mot, R., and Höfte, M. 2019. Pseudomonas cyclic lipopeptides suppress the rice blast fungus Magnaporthe oryzae by induced resistance and direct antagonism. Front. Plant Sci. 10:901.

Overbeek, R., Olson, R., Pusch, G. D., Olsen, G. J., Davis, J. J., Disz, T., Edwards, R. A., Gerdes, S., Parrello, B., and Shukla, M. 2014. The SEED and the rapid annotation of microbial genomes using subsystems technology (RAST). Nucleic Acids Res. 42: D206-D214.

Pruitt, K. D., Tatusova, T., Brown, G. R., and Maglott, D. R. 2011. NCBI reference sequences (RefSeq): Current status, new features and genome annotation policy. Nucleic Acids Res. 40:D130-D135.

Singh, A., Van Hamme, J. D., and Ward, O. P. 2007. Surfactants in microbiology and biotechnology: Part 2. Application aspects. Biotechnol. Adv. 25:99-121.

Wattam, A. R., Davis, J. J., Assaf, R., Boisvert, S., Brettin, T., Bun, C., Conrad, N., Dietrich, E. M., Disz, T., and Gabbard, J. L. 2017. Improvements to PATRIC, the allbacterial bioinformatics database and analysis resource center. Nucleic Acids Res. 45:D535-D542.

Wu, L., Xiao, W., Chen, G., Song, D., Khaskheli, M. A., Li, P., Zhang, S., and Feng, G. 2018. Identification of Pseudomonas mosselii BS011 gene clusters required for suppression of rice blast fungus Magnaporthe oryzae. J. Biotechnol. 282:1-9.

Zhang, Y., Chen, P., Ye, G., Lin, H., Ren, D., Guo, L., Zhu, B., and Wang, Z. 2019. Complete genome sequence of Pseudomonas parafulva PRS09-11288, a biocontrol strain produces the antibiotic phenazine-1-carboxylic acid. Curr. Microbiol. 76:1087-1091. 